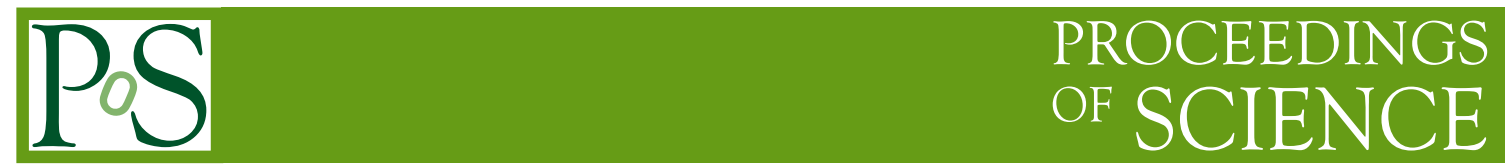

\title{
Higher dimensional HQET parameters
}

\section{Gil Paz*}

Department of Physics and Astronomy

Wayne State University

Detroit, Michigan 48201, USA

E-mail: gilpaz@wayne.edu

Effective field theories such as Heavy Quark Effective Theory (HQET) are indispensable tools in controlling the effects of the strong interaction. The increasing experimental precision requires the knowledge of higher dimensional operators. We present a general method that allows for an easy construction of HQET operators that contain two heavy quarks and any number of covariant derivatives.

9th International Workshop on the CKM Unitarity Triangle

28 November - 3 December 2016

Tata Institute for Fundamental Research (TIFR), Mumbai, India

* Speaker. 


\section{Motivation}

Inclusive semileptonic $B$ decays and the $Q_{7 \gamma}-Q_{7 \gamma}$ contribution to $B \rightarrow X_{s} \gamma$ can be described by a local operator product expansion: $\Gamma=\sum_{n=0}^{\infty} m_{b}^{-n} \sum_{k} c_{k, n}\left\langle O_{k, n}\right\rangle$ see the talk "Theory of radiative $B$ decays" in these proceedings. The Wilson coefficients $c_{k, n}$ are perturbative. The matrix elements $\left\langle O_{k, n}\right\rangle$ of Heavy Quark Effective Theory (HQET) operators are non-perturbative and often called HQET parameters. Higher dimensional HQET parameters are of phenomenological interest. For example, $\left|V_{c b}\right|$ extraction from inclusive $B$ decays [1] uses dimension 7 and 8 HQET operators [2].

Several questions arise. 1) Are these all the possible HQET operators at dimension 7 and 8 ? or do inclusive $B$ decays require a subset of the possible operators? 2) Can we construct higher dimensional HQET operators? 3) Since HQET and Non Relativistic QCD (NRQCD) are related $[3,4]$, what are the corresponding NRQCD operators? 4) What can we learn about the structure of effective field theories (EFTs)? We will answer all of these questions during this talk.

\section{A little bit of history}

Because of the relation between HQET, NRQCD, and Non Relativistic QED (NRQED), the question of possible operators at each dimension can be traced to the early days of quantum mechanics. The time and space components of the covariant derivative are $D_{t}=\partial / \partial t+i e A^{0}, \boldsymbol{D}=$ $\boldsymbol{\nabla}-i e \boldsymbol{A}$. Schrödinger equation contain the operator $i D_{t}+\boldsymbol{D}^{2} / 2 M$. When discussing hydrogen fine structure one encounters operators such as spin-orbit coupling: $\boldsymbol{\sigma} \cdot \boldsymbol{B}$, relativistic correction: $\boldsymbol{D}^{4}$, and the Darwin term: $\boldsymbol{\partial} \cdot \boldsymbol{E}$, where $\boldsymbol{E}=(-i / g)\left[D^{0}, \boldsymbol{D}\right]$ and $\boldsymbol{B}^{i}=\varepsilon^{i j k}(i / 2 g)\left[\boldsymbol{D}^{j}, \boldsymbol{D}^{k}\right]$. Organizing the operators in a Lagrangian form and keeping operators up to dimension 6 , one obtains the dimension-6 NRQED Lagrangian first presented in 1986 [5]:

$$
\mathscr{L}_{\mathrm{NRQED}}^{\operatorname{dim} \leq 6}=\psi^{\dagger}\left\{i D_{t}+\frac{\boldsymbol{D}^{2}}{2 M} c_{F} g \frac{\boldsymbol{\sigma} \cdot \boldsymbol{B}}{2 M}+c_{D} g \frac{[\boldsymbol{\partial} \cdot \boldsymbol{E}]}{8 M^{2}}+i c_{S} g \frac{\boldsymbol{\sigma} \cdot(\boldsymbol{D} \times \boldsymbol{E}-\boldsymbol{E} \times \boldsymbol{D})}{8 M^{2}}\right\} \psi .
$$

In 1994 the first systematic discussion of HQET parameters was presented in [6]. Between HQET fields $\bar{h} \ldots h$ the Dirac basis reduces to $\{1, \boldsymbol{\sigma}\}=\left\{1, s^{\lambda}\right\}$ with v.s $=0$, where $v$ is the velocity. The most general bilinear HQET operator is of the form $\bar{h} i D^{\mu_{1}} \ldots i D^{\mu_{n}}\left(s^{\lambda}\right) h$. This operator vanishes if it is contracted with $v_{\mu_{1}}, v_{\mu_{n}}$, or $v_{\lambda}$ [6]. Consider matrix elements of such operators between heavy meson states. One finds one dimension 3 operator with no derivatives: $\bar{h} h$ and dimension 4 operators with one derivatives: $\bar{h} i D^{\mu}\left(s^{\lambda}\right) h$ that have vanishing matrix elements. For dimension 5 and 6 one finds [6]

$$
\begin{aligned}
\left\langle H\left|\bar{h}\left(i D_{\alpha}\right)\left(i D_{\beta}\right) h\right| H\right\rangle & =2 M_{H}\left[g_{\alpha \beta}-v_{\alpha} v_{\beta}\right] \frac{1}{3} \lambda_{1} \\
\left\langle H\left|\bar{h}\left(i D_{\alpha}\right)\left(i D_{\beta}\right) s_{\lambda} h\right| H\right\rangle & =2 M_{H} d_{H} i \varepsilon_{v \alpha \beta \lambda} v^{v} \frac{1}{6} \lambda_{2} \\
\left\langle H\left|\bar{h}\left(i D_{\alpha}\right)\left(i D_{\mu}\right)\left(i D_{\beta}\right) h_{v}\right| H\right\rangle & =2 M_{H}\left[g_{\alpha \beta}-v_{\alpha} v_{\beta}\right] v_{\mu} \frac{1}{3} \rho_{1} \\
\left\langle H\left|\bar{h}\left(i D_{\alpha}\right)\left(i D_{\mu}\right)\left(i D_{\beta}\right) s_{\lambda} h\right| H\right\rangle & =2 M_{H} d_{H} i \varepsilon_{v \alpha \beta \lambda} v^{v} v_{\mu} \frac{1}{6} \rho_{2} .
\end{aligned}
$$

The same source also discussed higher dimensional operators, but unfortunately the enumeration of the operators is incorrect. 
For both dimension 5 and 6 we have two HQET parameters corresponding to two operators: a Spin-Independent (SI) operator and a Spin-Dependent (SD) operator. Notice that this is the same number of operator as in (2.1). This is not an accident. While HQET and NRQCD differ in their kinetic terms: $\mathscr{L}_{H Q E T}^{\text {kinetic }}=\bar{h} i v \cdot D h, \mathscr{L}_{N R Q C D}^{\text {kinetic }}=\psi^{\dagger}\left(i D_{t}+D^{2} / 2 M\right) \psi$, and power counting, there is a correspondence between HQET and NRQCD (NRQED) operators [3, 4]. For example:

$$
\text { NRQED (1920's-1980's) }
$$

$\begin{array}{cc}\text { Dimension 5 } & \boldsymbol{D}^{2} \\ \text { Dimension 6 } & \boldsymbol{\sigma} \cdot \boldsymbol{B} \\ & {[\boldsymbol{\partial} \cdot \boldsymbol{E}]} \\ & \boldsymbol{\sigma} \cdot(\boldsymbol{D} \times \boldsymbol{E}-\boldsymbol{E} \times \boldsymbol{D})\end{array}$

\section{HQET (1990’s)}

$$
\begin{gathered}
\left(i D_{\perp}\right)^{2} \\
\left(i D_{\perp}^{\mu}\right)\left(i D_{\perp}^{v}\right)\left(-i \sigma^{\mu v}\right) \\
\left(i D_{\perp \mu}\right)(i v \cdot D)\left(i D_{\perp}^{\mu}\right) \\
\left(i D_{\perp}^{\mu}\right)(i v \cdot D)\left(i D_{\perp}^{v}\right)\left(-i \sigma^{\mu v}\right)
\end{gathered}
$$

Table 1: Correspondence between dimension 5 and 6 HQET and NRQCD (NRQED) operators. The notation is $\sigma^{\mu v}=i\left[\gamma^{\mu}, \gamma^{v}\right] / 2$ and $D_{\perp}^{\mu}=D^{\mu}-v \cdot D v^{\mu}$.

In 1997 the NRQCD and HQET Lagrangian up to dimension 7 was given in [3]. The Lagrangian contains four SI and five SD operators. Two of the SD operators containing $\boldsymbol{\sigma} \cdot(\boldsymbol{B} \times \boldsymbol{B})$ and $\boldsymbol{\sigma} \cdot(\boldsymbol{E} \times \boldsymbol{E})$ vanish for NRQED.

The dimension 7 contribution to semileptonic decays was discussed in 2006 in [7] and again in 2010 in [2]. How many operators do we have at dimension 7? For SI operators [6] lists two, [3] lists four, [7] lists three, and [2] lists four. For SD operators [6] lists five, [3] lists five, [7] lists two, and [2] lists five. HQET-NRQCD correspondence implies four SI and five SD operators.

What about dimension 8 operators? [2] lists seven SI operators and eleven SD operators. In 2012 the dimension 8 NRQED Lagrangian was given in [8]. It lists four SI operators and eight SD operators. Comparing to [2] the operators not listed in [8] are presumably NRQCD operators that vanish for NRQED.

In all of the papers $[5,6,3,7,2,8]$ there is no derivation of the operators for each dimension. We learn from this history that finding all of the HQET and NRQCD operators at a given dimension is not easy. Is there a systematic way to do that?

\section{Higher dimensional HQET parameters}

As shown in [4] the answer is yes. We consider matrix elements of the form $\left\langle H\left|\bar{h} i D^{\mu_{1}} \ldots i D^{\mu_{n}} h\right| H\right\rangle$ and $\left\langle H\left|\bar{h} i D^{\mu_{1}} \ldots i D^{\mu_{n}} s^{\lambda} h\right| H\right\rangle$ and decompose them in terms of tensors $v^{\mu_{i}}, g^{\mu_{i} \mu_{j}}$, and $\varepsilon^{\alpha \beta \rho \sigma}$, subject to constraints from Parity and Time reversal symmetry $(P T)$, Hermitian conjugation, and the fact that we are working in four dimensions.

Parity and time reversal are symmetries of HQET. In particular under their combined operation we have $p=\left(p^{0}, \vec{p}\right) \stackrel{P T}{\rightarrow}\left(p^{0}, \vec{p}\right)=p \Rightarrow v=p / m \stackrel{P T}{\rightarrow} v, i D^{\mu} \stackrel{P T}{\rightarrow} i D^{\mu}, \bar{h} h \stackrel{P T}{\rightarrow} \bar{h} h$, and $\bar{h} s^{\lambda} h \stackrel{P T}{\rightarrow}-\bar{h} s^{\lambda} h$. Since $T$ is anti-linear we have in total

$$
\begin{array}{r}
\left\langle H\left|\bar{h} i D^{\mu_{1}} \ldots i D^{\mu_{n}} h\right| H\right\rangle \stackrel{P T}{=}\left\langle H\left|\bar{h} i D^{\mu_{1}} \ldots i D^{\mu_{n}} h\right| H\right\rangle^{*} \\
\left\langle H\left|\bar{h} i D^{\mu_{1}} \ldots i D^{\mu_{n}} s^{\lambda} h\right| H\right\rangle \stackrel{P T}{=}-\left\langle H\left|\bar{h} i D^{\mu_{1}} \ldots i D^{\mu_{n}} s^{\lambda} h\right| H\right\rangle^{*} .
\end{array}
$$


We conclude that matrix elements of SI operators are real and matrix elements of SD operators are complex.

Since $\bar{h} h, \bar{h} s^{\lambda} h, i D^{\mu}$ are hermitian, using Hermitian conjugation we find that

$$
\left\langle H\left|\bar{h} i D^{\mu_{1}} \ldots i D^{\mu_{n}}\left(s^{\lambda}\right) h\right| H\right\rangle=\left\langle H\left|\left(\bar{h} i D^{\mu_{1}} \ldots i D^{\mu_{n}}\left(s^{\lambda}\right) h\right)^{\dagger}\right| H\right\rangle^{*}=\left\langle H\left|\bar{h} i D^{\mu_{n}} \ldots i D^{\mu_{1}}\left(s^{\lambda}\right) h\right| H\right\rangle^{*} .
$$

Combining this with the $P T$ constraints we find that under inversion of the indices matrix elements of SI operators are symmetric and matrix elements of SD operators are anti-symmetric.

$H$ is a pseudo-scalar so the matrix element can only depend on the tensor $v^{\mu_{i}}, g^{\mu_{i} \mu_{j}}$, and $\varepsilon^{\alpha \beta \rho \sigma}$. Alternatively following [2] we define $\Pi^{\mu v}=g^{\mu v}-v^{\mu} v^{v}$. For the standard choice of $v=(1,0,0,0)$ : $\Pi^{00}=0$ and $\Pi^{i j}=-\delta^{i j}$. Since the indices in $\varepsilon^{\alpha \beta \rho \sigma}$ cannot all be orthogonal to $v$, we can replace $\varepsilon^{\alpha \beta \rho \sigma}$ by $\varepsilon^{\alpha \beta \rho \sigma} v_{\alpha}$. In the following we will decompose the matrix elements in term of $v^{\mu_{i}}, \Pi^{\mu_{i} \mu_{j}}$, and $\varepsilon^{\alpha \beta \rho \sigma} v_{\alpha}$.

Another constraint arises from the fact that we are working in four dimensions. As a result not all tensors with more than four indices are independent. For example for the dimension 7 SD HQET operators we need the tensor $\Pi^{\mu v} \varepsilon^{\alpha \beta \rho \sigma} v_{\alpha}$. Three of its indices are the same and tensors obtained by permuting its indices are not linearly independent. Analogous constraint for SI operators start at dimension 11 where we need $\Pi^{\mu_{1} \mu_{2}} \Pi^{\mu_{3} \mu_{4}} \Pi^{\mu_{5} \mu_{6}} \Pi^{\mu_{7} \mu_{8}}$. Since all of the indices are space-like, we must have four identical indices.

Using these general consideration one can list the various HQET parameters that appear in the decomposition of the general HQET operator of a given dimension. The dimension 5 and 6 decompositions are listed in (2.2). Let us find the decomposition of the dimension 7 SI operator. The matrix element is $\left\langle H\left|\bar{h} i D^{\mu_{1}} i D^{\mu_{2}} i D^{\mu_{3}} i D^{\mu_{4}} h\right| H\right\rangle$. Consider its decomposition in terms possible tensors. We can have a product of two $\Pi$ 's or a product of $\Pi$ and two $v$ 's. For products of two $\Pi$ 's we can contract $\mu_{1}$ with $\mu_{2}, \mu_{3}$, or $\mu_{4}$ using $\Pi$. The other two indices are also contracted by $\Pi$. In total we have three such combinations of two ח's. Using two $v$ 's, they can only be contracted with $\mu_{2}$ and $\mu_{3}$ giving us a fourth tensor. In total we have

$$
\begin{aligned}
\frac{1}{2 M_{H}}\left\langle H\left|\bar{h} i D^{\mu_{1}} i D^{\mu_{2}} i D^{\mu_{3}} i D^{\mu_{4}} h\right| H\right\rangle & =a_{12}^{(7)} \Pi^{\mu_{1} \mu_{2}} \Pi^{\mu_{3} \mu_{4}}+a_{13}^{(7)} \Pi^{\mu_{1} \mu_{3}} \Pi^{\mu_{2} \mu_{4}}+ \\
& +a_{14}^{(7)} \Pi^{\mu_{1} \mu_{4}} \Pi^{\mu_{2} \mu_{3}}+b^{(7)} \Pi^{\mu_{1} \mu_{4}} v^{\mu_{2}} v^{\mu_{3}}
\end{aligned}
$$

Similarly one can find the decomposition of the SD operator:

$$
\begin{aligned}
& \frac{1}{2 M_{H}}\left\langle H\left|\bar{h} i D^{\mu_{1}} i D^{\mu_{2}} i D^{\mu_{3}} i D^{\mu_{4}} s^{\lambda} h\right| H\right\rangle=i \tilde{a}_{12}^{(7)}\left(\Pi^{\mu_{1} \mu_{2}} \varepsilon^{\rho \mu_{3} \mu_{4} \lambda} v_{\rho}-\Pi^{\mu_{4} \mu_{3}} \varepsilon^{\rho \mu_{2} \mu_{1} \lambda} v_{\rho}\right)+ \\
& +i \tilde{a}_{13}^{(7)}\left(\Pi^{\mu_{1} \mu_{3}} \varepsilon^{\rho \mu_{2} \mu_{4} \lambda} v_{\rho}-\Pi^{\mu_{4} \mu_{2}} \varepsilon^{\rho \mu_{3} \mu_{1} \lambda} v_{\rho}\right)+i \tilde{a}_{14}^{(7)} \Pi^{\mu_{1} \mu_{4}} \varepsilon^{\rho \mu_{2} \mu_{3} \lambda} v_{\rho}+ \\
+ & i \tilde{a}_{23}^{(7)} \Pi^{\mu_{2} \mu_{3}} \varepsilon^{\rho \mu_{1} \mu_{4} \lambda} v_{\rho}+i \tilde{b}^{(7)} v^{\mu_{2}} v^{\mu_{3}} \varepsilon^{\rho \mu_{1} \mu_{4} \lambda} v_{\rho} .
\end{aligned}
$$

The notation for the parameters is such that the subscript denotes the first two indices that are contracted via $\Pi$ 's in numerical order, and the dimension of the operators appears in the superscript. How many HQET operators do we have at dimension 7? the answer is four SI operators and five SD operators confirming [3] and [2]. 
The dimension 8 decomposition is

$$
\begin{aligned}
& \frac{1}{2 M_{H}}\left\langle H\left|\bar{h} i D^{\mu_{1}} i D^{\mu_{2}} i D^{\mu_{3}} i D^{\mu_{4}} i D^{\mu_{5}} h\right| H\right\rangle=a_{12}^{(8)}\left(\Pi^{\mu_{1} \mu_{2}} \Pi^{\mu_{3} \mu_{5}} v^{\mu_{4}}+\Pi^{\mu_{1} \mu_{3}} \Pi^{\mu_{4} \mu_{5}} v^{\mu_{2}}\right)+ \\
& a_{13}^{(8)}\left(\Pi^{\mu_{1} \mu_{3}} \Pi^{\mu_{2} \mu_{5}} v^{\mu_{4}}+\Pi^{\mu_{3} \mu_{5}} \Pi^{\mu_{1} \mu_{4}} v^{\mu_{2}}\right)+a_{15}^{(8)}\left(\Pi^{\mu_{1} \mu_{5}} \Pi^{\mu_{3} \mu_{4}} v^{\mu_{2}}+\Pi^{\mu_{1} \mu_{5}} \Pi^{\mu_{2} \mu_{3}} v^{\mu_{4}}\right)+ \\
& b_{12}^{(8)} \Pi^{\mu_{1} \mu_{2}} \Pi^{\mu_{4} \mu_{5}} v^{\mu_{3}}+b_{14}^{(8)} \Pi^{\mu_{1} \mu_{4}} \Pi^{\mu_{2} \mu_{5}} v^{\mu_{3}}+b_{15}^{(8)} \Pi^{\mu_{1} \mu_{5}} \Pi^{\mu_{2} \mu_{4}} v^{\mu_{3}}+c^{(8)} \Pi^{\mu_{1} \mu_{5}} v^{\mu_{2}} v^{\mu_{3}} v^{\mu_{4}} \\
& \frac{1}{2 M_{H}}\left\langle H\left|\bar{h} i D^{\mu_{1}} i D^{\mu_{2}} i D^{\mu_{3}} i D^{\mu_{4}} i D^{\mu_{5}} s^{\lambda} h\right| H\right\rangle=i \tilde{a}_{12}^{(8)}\left(v^{\mu_{3}} \Pi^{\mu_{1} \mu_{2}} \varepsilon^{\rho \mu_{4} \mu_{5} \lambda} v_{\rho}-v^{\mu_{3}} \Pi^{\mu_{4} \mu_{5}} \varepsilon^{\rho \mu_{2} \mu_{1} \lambda} v_{\rho}\right) \\
& +i \tilde{a}_{14}^{(8)}\left(v^{\mu_{3}} \Pi^{\mu_{1} \mu_{4}} \varepsilon^{\rho \mu_{2} \mu_{5} \lambda} v_{\rho}-v^{\mu_{3}} \Pi^{\mu_{5} \mu_{2}} \varepsilon^{\rho \mu_{4} \mu_{1} \lambda} v_{\rho}\right)+i \tilde{a}_{15}^{(8)} v^{\mu_{3}} \Pi^{\mu_{1} \mu_{5}} \varepsilon^{\rho \mu_{2} \mu_{4} \lambda} v_{\rho}+i \tilde{a}_{24}^{(8)} v^{\mu_{3}} \Pi^{\mu_{2} \mu_{4}} \varepsilon^{\rho \mu_{1} \mu_{5} \lambda} v_{\rho} \\
& +i \tilde{b}_{13}^{(8)}\left(v^{\mu_{2}} \Pi^{\mu_{1} \mu_{3}} \varepsilon^{\rho \mu_{4} \mu_{5} \lambda} v_{\rho}-v^{\mu_{4}} \Pi^{\mu_{5} \mu_{3}} \varepsilon^{\rho \mu_{2} \mu_{1} \lambda} v_{\rho}\right)+i \tilde{b}_{14}^{(8)}\left(v^{\mu_{2}} \Pi^{\mu_{1} \mu_{4}} \varepsilon^{\rho \mu_{3} \mu_{5} \lambda} v_{\rho}-v^{\mu_{4}} \Pi^{\mu_{5} \mu_{2}} \varepsilon^{\rho \mu_{3} \mu_{1} \lambda} v_{\rho}\right)+ \\
& +i \tilde{b}_{15}^{(8)}\left(v^{\mu_{2}} \Pi^{\mu_{1} \mu_{5}} \varepsilon^{\rho \mu_{3} \mu_{4} \lambda} v_{\rho}-v^{\mu_{4}} \Pi^{\mu_{1} \mu_{5}} \varepsilon^{\rho \mu_{3} \mu_{2} \lambda} v_{\rho}\right)+i \tilde{b}_{34}^{(8)}\left(v^{\mu_{2}} \Pi^{\mu_{3} \mu_{4}} \varepsilon^{\rho \mu_{1} \mu_{5} \lambda} v_{\rho}-v^{\mu_{4}} \Pi^{\mu_{3} \mu_{2}} \varepsilon^{\rho \mu_{5} \mu_{1} \lambda} v_{\rho}\right)+ \\
& +i \tilde{b}_{35}^{(8)}\left(v^{\mu_{2}} \Pi^{\mu_{3} \mu_{5}} \varepsilon^{\rho \mu_{1} \mu_{4} \lambda} v_{\rho}-v^{\mu_{4}} \Pi^{\mu_{3} \mu_{1}} \varepsilon^{\rho \mu_{5} \mu_{2} \lambda} v_{\rho}\right)+i \tilde{b}_{45}^{(8)}\left(v^{\mu_{2}} \Pi^{\mu_{4} \mu_{5}} \varepsilon^{\rho \mu_{1} \mu_{3} \lambda} v_{\rho}-v^{\mu_{4}} \Pi^{\mu_{2} \mu_{1}} \varepsilon^{\rho \mu_{5} \mu_{3} \lambda} v_{\rho}\right)+ \\
& +i \tilde{c}^{(8)} v^{\mu_{2}} v^{\mu_{3}} v^{\mu_{4}} \varepsilon^{\rho \mu_{1} \mu_{5} \lambda} v_{\rho} \text {. }
\end{aligned}
$$

How many HQET operators do we have at dimension 8? The answer is seven SI operators and eleven SD operators confirming [2]. Similarly one can find the dimension 8 NRQCD operators that vanish for NRQED. These are given in [4].

We can go beyond known results in the literature. For example, there are 24 possible dimension 9 HQET operators [4]:

$$
\begin{aligned}
& \frac{1}{2 M_{H}}\left\langle H\left|\bar{h} i D^{\mu_{1}} i D^{\mu_{2}} i D^{\mu_{3}} i D^{\mu_{4}} i D^{\mu_{5}} i D^{\mu_{6}} h\right| H\right\rangle=a_{12,34}^{(9)} \Pi^{\mu_{1} \mu_{2}} \Pi^{\mu_{3} \mu_{4}} \Pi^{\mu_{5} \mu_{6}}+ \\
& +a_{12,35}^{(9)}\left(\Pi^{\mu_{1} \mu_{2}} \Pi^{\mu_{3} \mu_{5}} \Pi^{\mu_{4} \mu_{6}}+\Pi^{\mu_{1} \mu_{3}} \Pi^{\mu_{2} \mu_{4}} \Pi^{\mu_{5} \mu_{6}}\right)+a_{12,36}^{(9)}\left(\Pi^{\mu_{1} \mu_{2}} \Pi^{\mu_{3} \mu_{6}} \Pi^{\mu_{4} \mu_{5}}+\Pi^{\mu_{1} \mu_{4}} \Pi^{\mu_{2} \mu_{3}} \Pi^{\mu_{5} \mu_{6}}\right)+ \\
& +a_{13,25}^{(9)} \Pi^{\mu_{1} \mu_{3}} \Pi^{\mu_{2} \mu_{5}} \Pi^{\mu_{4} \mu_{6}}+a_{13,26}^{(9)}\left(\Pi^{\mu_{1} \mu_{3}} \Pi^{\mu_{2} \mu_{6}} \Pi^{\mu_{4} \mu_{5}}+\Pi^{\mu_{1} \mu_{5}} \Pi^{\mu_{2} \mu_{3}} \Pi^{\mu_{4} \mu_{6}}\right)+a_{14,25}^{(9)} \Pi^{\mu_{1} \mu_{4}} \Pi^{\mu_{2} \mu_{5}} \Pi^{\mu_{3} \mu_{6}}+ \\
& +a_{14,26}^{(9)}\left(\Pi^{\mu_{1} \mu_{4}} \Pi^{\mu_{2} \mu_{6}} \Pi^{\mu_{3} \mu_{5}}+\Pi^{\mu_{1} \mu_{5}} \Pi^{\mu_{2} \mu_{4}} \Pi^{\mu_{3} \mu_{6}}\right)+a_{15,26}^{(9)} \Pi^{\mu_{1} \mu_{5}} \Pi^{\mu_{2} \mu_{6}} \Pi^{\mu_{3} \mu_{4}}+a_{16,23}^{(9)} \Pi^{\mu_{1} \mu_{6}} \Pi^{\mu_{2} \mu_{3}} \Pi^{\mu_{4} \mu_{5}} \\
& +a_{16,24}^{(9)} \Pi^{\mu_{1} \mu_{6}} \Pi^{\mu_{2} \mu_{4}} \Pi^{\mu_{3} \mu_{5}}+a_{16,25}^{(9)} \Pi^{\mu_{1} \mu_{6}} \Pi^{\mu_{2} \mu_{5}} \Pi^{\mu_{3} \mu_{4}}+b_{12,36}^{(9)}\left(\Pi^{\mu_{1} \mu_{2}} \Pi^{\mu_{3} \mu_{6}} v^{\mu_{4}} v^{\mu_{5}}+\Pi^{\mu_{1} \mu_{4}} \Pi^{\mu_{5} \mu_{6}} v^{\mu_{2}} v^{\mu_{3}}\right)+ \\
& +b_{12,46}^{(9)}\left(\Pi^{\mu_{1} \mu_{2}} \Pi^{\mu_{4} \mu_{6}} v^{\mu_{3}} v^{\mu_{5}}+\Pi^{\mu_{1} \mu_{3}} \Pi^{\mu_{5} \mu_{6}} v^{\mu_{2}} v^{\mu_{4}}\right)+b_{12,56}^{(9)} \Pi^{\mu_{1} \mu_{2}} \Pi^{\mu_{5} \mu_{6}} v^{\mu_{3}} v^{\mu_{4}}+ \\
& +b_{13,26}^{(9)}\left(\Pi^{\mu_{1} \mu_{3}} \Pi^{\mu_{2} \mu_{6}} v^{\mu_{4}} v^{\mu_{5}}+\Pi^{\mu_{1} \mu_{5}} \Pi^{\mu_{4} \mu_{6}} v^{\mu_{2}} v^{\mu_{3}}\right)+b_{13,46}^{(9)} \Pi^{\mu_{1} \mu_{3}} \Pi^{\mu_{4} \mu_{6}} v^{\mu_{2}} v^{\mu_{5}}+ \\
& +b_{14,26}^{(9)}\left(\Pi^{\mu_{1} \mu_{4}} \Pi^{\mu_{2} \mu_{6}} v^{\mu_{3}} v^{\mu_{5}}+\Pi^{\mu_{1} \mu_{5}} \Pi^{\mu_{3} \mu_{6}} v^{\mu_{2}} v^{\mu_{4}}\right)+b_{14,36}^{(9)} \Pi^{\mu_{1} \mu_{4}} \Pi^{\mu_{3} \mu_{6}} v^{\mu_{2}} v^{\mu_{5}}+b_{15,26}^{(9)} \Pi^{\mu_{1} \mu_{5}} \Pi^{\mu_{2} \mu_{6}} v^{\mu_{3}} v^{\mu_{4}}+ \\
& b_{16,23}^{(9)}\left(\Pi^{\mu_{1} \mu_{6}} \Pi^{\mu_{2} \mu_{3}} v^{\mu_{4}} v^{\mu_{5}}+\Pi^{\mu_{1} \mu_{6}} \Pi^{\mu_{4} \mu_{5}} v^{\mu_{2}} v^{\mu_{3}}\right)+b_{16,24}^{(9)}\left(\Pi^{\mu_{1} \mu_{6}} \Pi^{\mu_{2} \mu_{4}} v^{\mu_{3}} v^{\mu_{5}}+\Pi^{\mu_{1} \mu_{6}} \Pi^{\mu_{3} \mu_{5}} v^{\mu_{2}} v^{\mu_{4}}\right)+ \\
& +b_{16,25}^{(9)} \Pi^{\mu_{1} \mu_{6}} \Pi^{\mu_{2} \mu_{5}} v^{\mu_{3}} v^{\mu_{4}}+b_{16,34}^{(9)} \Pi^{\mu_{1} \mu_{6}} \Pi^{\mu_{3} \mu_{4}} v^{\mu_{2}} v^{\mu_{5}}+c^{(9)} \Pi^{\mu_{1} \mu_{6}} v^{\mu_{2}} v^{\mu_{3}} v^{\mu_{4}} v^{\mu_{5}}
\end{aligned}
$$

An interesting question is what are the Wilson coefficients of the operators. In particular what are the relations between coefficients of operators of different dimensions. These are known as "reparameterization invariance" [10] or "Lorentz invariance" constraints [11]. For NRQED such relations are known for up to dimension 8 operators $[11,8]$ but not for NRQCD or HQET operators above dimension 6. Such relations allow to determine the contribution of a certain higher dimensional operators based on the knowledge of lower dimensional operators. This has applications to semileptonic and radiative $B$ decays, see e.g. [12, 13, 14]. 


\section{Conclusions}

We presented a general method to construct HQET operators by using the tensor decomposition of HQET matrix elements. There are several applications to this method. First, the tensor decomposition allows to easily relate different bases ${ }^{1}$. In [4] we relate it to the dimension 7 operators basis of [3] and [2] and the dimension 8 operators basis of [2]. Second, we present for the first time the decomposition of the general SI dimension 9 HQET operator, equation (3.7). Third, in [4] moments of the leading power shape function are calculated up to and including dimension 9 HQET operators. This can improve the parameterization of the shape function relevant to the extraction of $\left|V_{u b}\right|$. Fourth, in [4] we present the full dimension 8 NRQCD Lagrangian.

We can now answer the questions posed earlier. 1) Are the dimension 7 and 8 HQET operators in [2] all the possible operators? Yes. 2) Can we construct higher dimensional HQET operators? Yes, we presented the general method for that. 3) What are the corresponding NRQCD operators? The answer is given in [4]. 4) What can we learn about the structure of EFTs? It seems to be simpler than we might think. This is also the conclusion of [15] for the Standard Model EFT.

\section{Acknowledgments}

This work was supported by DOE grant DE-SC0007983.

\section{References}

[1] P. Gambino, K. J. Healey and S. Turczyk, Phys. Lett. B 763, 60 (2016) [arXiv:1606.06174 [hep-ph]].

[2] T. Mannel, S. Turczyk and N. Uraltsev, JHEP 1011, 109 (2010) [arXiv:1009.4622 [hep-ph]].

[3] A. V. Manohar, Phys. Rev. D 56, 230 (1997) [hep-ph/9701294].

[4] A. Gunawardana and G. Paz, arXiv:1702.08904 [hep-ph].

[5] W. E. Caswell and G. P. Lepage, Phys. Lett. 167B, 437 (1986).

[6] T. Mannel, Phys. Rev. D 50, 428 (1994) [hep-ph/9403249].

[7] B. M. Dassinger, T. Mannel and S. Turczyk, JHEP 0703, 087 (2007) [hep-ph/0611168].

[8] R. J. Hill, G. Lee, G. Paz and M. P. Solon, Phys. Rev. D 87, 053017 (2013) [arXiv:1212.4508 [hep-ph]].

[9] J. Heinonen and T. Mannel, arXiv:1609.01334 [hep-ph].

[10] M. E. Luke and A. V. Manohar, Phys. Lett. B 286, 348 (1992) [hep-ph/9205228].

[11] J. Heinonen, R. J. Hill and M. P. Solon, Phys. Rev. D 86, 094020 (2012) [arXiv:1208.0601 [hep-ph]].

[12] T. Becher, H. Boos and E. Lunghi, JHEP 0712, 062 (2007) [arXiv:0708.0855 [hep-ph]].

[13] T. Ewerth, P. Gambino and S. Nandi, Nucl. Phys. B 830, 278 (2010) [arXiv:0911.2175 [hep-ph]].

[14] A. V. Manohar, Phys. Rev. D 82, 014009 (2010) [arXiv:1005.1952 [hep-ph]].

[15] B. Henning, X. Lu, T. Melia and H. Murayama, arXiv:1512.03433 [hep-ph].

\footnotetext{
${ }^{1}$ The appendix of [9] also lists a tensor decomposition for HQET operators up to dimension 8 and relates it to [2].
} 